\title{
The accuracy of presepsin for the diagnosis of sepsis from SIRS: a systematic review and meta-analysis
}

\author{
Zhongjun Zheng ${ }^{\dagger}$, Libing Jiang ${ }^{\dagger}$, Ligang Ye ${ }^{\dagger}$, Yuzhi Gao, Luping Tang and Mao Zhang ${ }^{*}$
}

\begin{abstract}
Background: Sepsis is a common condition that has a high mortality and morbidity that need prompt diagnosis and treatment. Biomarkers like Soluble CD14 subtype (sCD14-ST, presepsin) may be useful in identifying patients with sepsis and its diagnostic superiority has been confirmed by several preliminary studies. The aim of this study was systematically and quantitatively to evaluate the value of presepsin for the diagnosis of sepsis through the method of meta-analysis.
\end{abstract}

Methods: Four major databases, including MEDLINE, EMBASE, ISI Web of Knowledge, and the Cochrane Library were systematically searched from inception to March 2015. Two investigators conducted the processes of literature search, study selection, data extraction, and quality evaluation independently. And the original data were extracted from all eligible individual studies to construct two-by-two tables.

Results: A total of eight studies comprising 1757 patients were included in this meta-analysis. The pooled sensitivity, specificity, and diagnostic odds ratio were 0.77 (95\% confidence interval [Cl]: 0.75-0.80), 0.73 (95\% Cl 0.69-0.77), and 14.25 (95\% Cl 8.66-23.42), respectively. The summary receiver operating characteristic curve (SROC) area under the curve (AUC) was 0.8598 . The subgroup analysis based on excluding the outliers showed that the pooled sensitivity and specificity were 0.85 (95\% Cl 0.81-0.89) and 0.65 (95\% Cl 0.59-0.70), respectively. The AUC was 0.8213 with no significant heterogeneity.

Conclusions: Presepsin has moderate diagnostic capacity for the detection of sepsis. Further research of presepsin is needed before widespread use in emergency department. And presepsin in combination with other laboratory biomarkers in diagnosing sepsis may be the focus of future studies.

Keywords: Sepsis, SIRS, Presepsin, SCD14-ST

\section{Background}

Sepsis is a condition in which the immune system overreacts to infection, releasing inflammatory mediators into the peripheral blood and triggering widespread inflammation. Early detection and timely treatment of the above pathologic processes may be able to prevent the occurrence of multiple organ dysfunction (MODS)

\footnotetext{
*Correspondence: zmhzze@163.com

${ }^{\dagger}$ Zhongjun Zheng, Libing Jiang, and Ligang Ye contributed equally to this work

Department of Emergency Medicine, Second Affiliated Hospital, School of Medicine and Institute of Emergency Medicine, Zhejiang University, No 88, Jiefang Rd, Hangzhou 310009, China
}

induced by severe sepsis and septic shock. Sepsis occurs in 1-2 \% of all hospitalizations, and it is the leading cause of mortality in critically ill patients [1,2]. Despite modern antibiotic therapy in combined with cardiovascular and respiratory support, mortality rates still remain between 30 and $60 \%$ [3-5]. Therefore, early diagnosis and treatment are hot topic among intensivists [5].

However, rapid and accurate diagnosis of sepsis is often difficult in routine clinical practice because the clinical manifestations of this condition can overlap with many non-infectious causes of systemic inflammation, such as pancreatitis, ischemia, multiple trauma, and hemorrhagic shock, which are collectively 
termed a systemic inflammatory response syndrome (SIRS) [6]. Microbiological culture is considered as the reference standard in diagnosing infectious condition, whereas it usually needs several days to obtain the results [7].

Presepsin, also named soluble cluster-of-differentiation 14 subtype ( $\mathrm{SCD} 14-\mathrm{ST}$ ), is a $13 \mathrm{kDa}$ protein that is a truncated N-terminal fragment of CD14 [8]. CD14 is a high-affinity receptor for complexes of lipopolysaccharide (LPS) and LPS-binding proteins (LPB), which is a multifunction cell surface glycoprotein expressed on the surface of various cells including monocytes, macrophages, neutrophils, and B cells $[9,10]$. By shedding of CD14 from the cell membrane and releasing into circulation during infection status, the LPS-LPBCD14 complex yields soluble CD14. Then, sCD14 is activated by plasma proteases during the circulating process, and thus generates a $13 \mathrm{kDa}$ protein named sCD14-ST or presepsin. Presepsin increases significantly in the blood of sepsis patients, and it has been studied as a marker to differentiate sepsis from other non-infectious causes of SIRS [8]. The results of several early studies were encouraging, and it has been reported that the diagnostic value of presepsin was superior to procalcitonin (PCT) and C-reactive protein (CRP) [11, 12]. However, few studies produced disappointing results $[13,14]$. Meanwhile, many studies included patients who did not have SIRS. This may add further uncertainty in assessing the diagnostic accuracy of presepsin. Thus, the aim of our study was to systematically explore the diagnostic value of presepsin in patients with sepsis.

\section{Methods}

The present meta-analysis was conducted and reported according to the Preferred Reporting Items for Systematic Reviews and Meta-analyses Statement (PRISMA).

\section{Data sources}

MEDLINE, Excerpta Medica database (EMBASE), ISI Web of Knowledge, and Cochrane Library databases were searched from the inception to March 2015. The following keywords or medical subject headings $(\mathrm{MeSH})$ were used: "presepsin" or "sCD14-ST" or "soluble CD-14 subtype" or "soluble cluster of differentiation 14 subtype" and "sepsis" or "SIRS" or "systemic inflammatory response syndrome" or "infection." We also searched the abstracts that were presented at the annual meetings of the American College of Emergency Physicians, the Society of Critical Care Medicine, and the Society for Academic Emergency Medicine. The reference lists of eligible articles and related reviews were also screened to identify further studies.

\section{Eligibility criteria}

Two reviewers (ZJZ) and (LBJ) independently evaluated the studies for their eligibility to be included. In cases of disagreement, a consensus was reached by discussion or by consultation to a third reviewer (LGY). Studies were considered eligible if the following criteria were met: providing the presepsin concentrations of sepsis patients and non-sepsis patients; having sufficient data to construct the $2 \times 2$ contingency table; having a well-defined reference standard about diagnosing sepsis(defined by the American College of Chest Physicians/Society of Critical Care Medicine Consensus Conference, ACCP/SCCM) [15].

Reviews, correspondence, editorials, and conference abstracts were excluded. Studies with the same authors were cautiously investigated. Studies were also excluded if they were limited to restrictive subgroups, such as some special types of sepsis like burn sepsis. However, their reference lists were screened to identify further studies for inclusion.

\section{Data extraction}

The following items were extracted using a specific sheet which was constructed in advance: authors and year of study, the country, study design, patient setting, number and characteristics of patients, presepsin measuring instrument, cut-off point, prevalence of sepsis, diagnostic sensitivity and specificity, inclusion criteria, and reference standard. Two reviewers independently extracted the data from each study. Disagreements were resolved by consensus.

\section{Quality assessment}

The methodological quality of all individual study was assessed using the QUADAS-2 tool [16], which was recommended by the Cochrane handbook for diagnostic test accuracy reviews. The QUADAS-2 tool comprises four domains: patient selection, index test, reference standard, and flow and timing. Each domain is assessed in terms of risk of bias, and the first three domains are also assessed in terms of concerns regarding applicability. Signaling questions are included to help judge the risk of bias of each study. The assessment was performed independently by two reviewers (ZJZ, LBJ). Disagreements were resolved by consensus.

\section{Statistical analysis}

True positive (TP), false positive (FP), false negative (FN), and true negative (TN) were obtained from each study. Sensitivity and specificity with its $95 \%$ confidence were calculated from the $2 \times 2$ contingency table of each study. We added $1 / 2$ to all cells of the studies with zero. Meanwhile, the meta-analysis was performed by calculating the pooled sensitivity, specificity, positive likelihood ratio (LR), negative LR, and diagnostic odds ratios (OR). 
Pooled results were constructed by using either the fixedeffect model when significant heterogeneity was absent or the random-effect model when significant heterogeneity was present [17].

Heterogeneity of the included studies was explored using the Cochrane Q test. Inconsistency $\left(I^{2}\right)$ expresses the variability attributable to heterogeneity across the studies in the form of a percentage. The spearman coefficient between the logit of sensitivity and logit of 1-specificity was performed to test threshold effect and a strong positive correlation indicates threshold effect [18]. There were several other factors that might contribute to the heterogeneity, including patient setting, characteristics of patients, presepsin measuring instrument, inclusion criteria, and reference standard [19]. $I^{2}>50 \%$ or $P<0.05$ suggested the presence of significant heterogeneity among included studies [20]. If substantial heterogeneity was found, the meta-regression techniques would be used to explore the reasons for the heterogeneity. Metaregression was made using a generalization of Littenberg and Moses Linear model. The model was weighted by inverse of the variance or study size [18, 19]. In addition, subgroup analyses were performed according to the result of meta-regression.

Finally, if the studies were reasonably homogeneous, an AUC was calculated. The closer that the value of the area under the curve is to 1 , the better validated the diagnostic test is. What's more, we used a $Q^{*}$ point from the SROC curve to obtain the maximum joint sensitivity and specificity. The $Q^{*}$ point is the intersection between a symmetrical SROC curve and the antidiagonal line, at which sensitivity equals specificity. Comparing to other parameters, $Q^{*}$ point is a single-number summarizing of the test performance and has the advantage of being less affected by heterogeneity $[18,21]$.

Publication bias was tested by Deeks' asymmetry test and a funnel plot. The slope coefficient with $P<0.05$ indicated the presence of publication bias.

All analyses including the pooling of sensitivity, specificity, positive LR, negative LR, diagnostic OR, SROC curve, and meta-regression were conducted using freeware Meta-Disc, version 1.4 (Ramon Y Cajal Hospital, Madrid, Spain) [22]. Study quality was performed using Review Manager 5.3 (Oxford, UK: The Cochrane Collaboration). Publication bias was performed using Stata version 12.0 (Stata Corporation, College Station, Tex). By convention, $P<0.05$ was considered statistically significant.

\section{Results}

\section{Identification of studies}

The flow diagram of study selection is shown in Fig. 1. A total of 135 records were retrieved by searching the databases of MEDLINE, EMBASE, ISI Web of Knowledge, and Cochrane Library databases. After removing the 14 duplicates, the titles and abstracts of the remaining 121 records were screened. Then, 61 apparently irrelevant studies were excluded, and 60 potentially relevant articles were identified for further review.

After full-text review, 52 articles were excluded: 31 were Conference abstracts, four were review articles [23-26], two were correspondence letters [8,27], one was editorial [28], three studied on neonates and had different reference standards [29-31], three did not have sufficient data to reconstruct the $2 \times 2$ contingency table [ 9 , $32,33]$, one had no SIRS patients in control group [34], one had health people in control group and it had different inclusion and exclusion criteria compared to our criteria [35], three had too narrow a spectrum of patients [36-38], one was about other infectious disease [39], and two studies were designed of self pre- and post-control observation [40, 41].

Finally, eight studies fulfilled the inclusion criteria and were eligible for our meta-analysis $[7,12,13,42-46]$. The characteristics of all eight studies included in our analysis are shown in Table 1. A total of 1757 patients were included in our meta-analysis, of which, 1240 were emergency department visits. The prevalence of sepsis ranged from 16.37 to $85.11 \%$. All the studies used a compact automated immune analyzer PATHFAST (Japan or Germany), which is based on a chemiluminescent enzyme immunoassay, to determine the plasma presepsin concentrations. The test threshold of presepsin of diagnosing sepsis ranged from 317 to $729 \mathrm{pg} / \mathrm{ml}$.

\section{Quality of studies}

Details of the methodological assessment are shown in Fig. 2. We evaluated the quality of the eight included studies using the QUADAS-2 tool. According to the results of methodological assessment, all included studies possessed acceptable quality. The ACCP/SCCM guideline was used to diagnose sepsis in all studies, and the same index test criterion was applied to each patient. Meanwhile, all of them used a prospective study design. However, all the studies did not pre-specify a threshold except one [46]. There is no evidence that the reference standard results of the studies interpreted without knowledge of the results of the index test except one [44].

\section{Heterogeneity exploration and data synthesis}

In the exploration of heterogeneity, the Spearman correlation coefficient between the logit of sensitivity and logit of 1 -specificity was $0.190(P=0.651)$ with the slope of the regression line near zero, suggesting that there was no significant threshold effect present among the eight studies. 


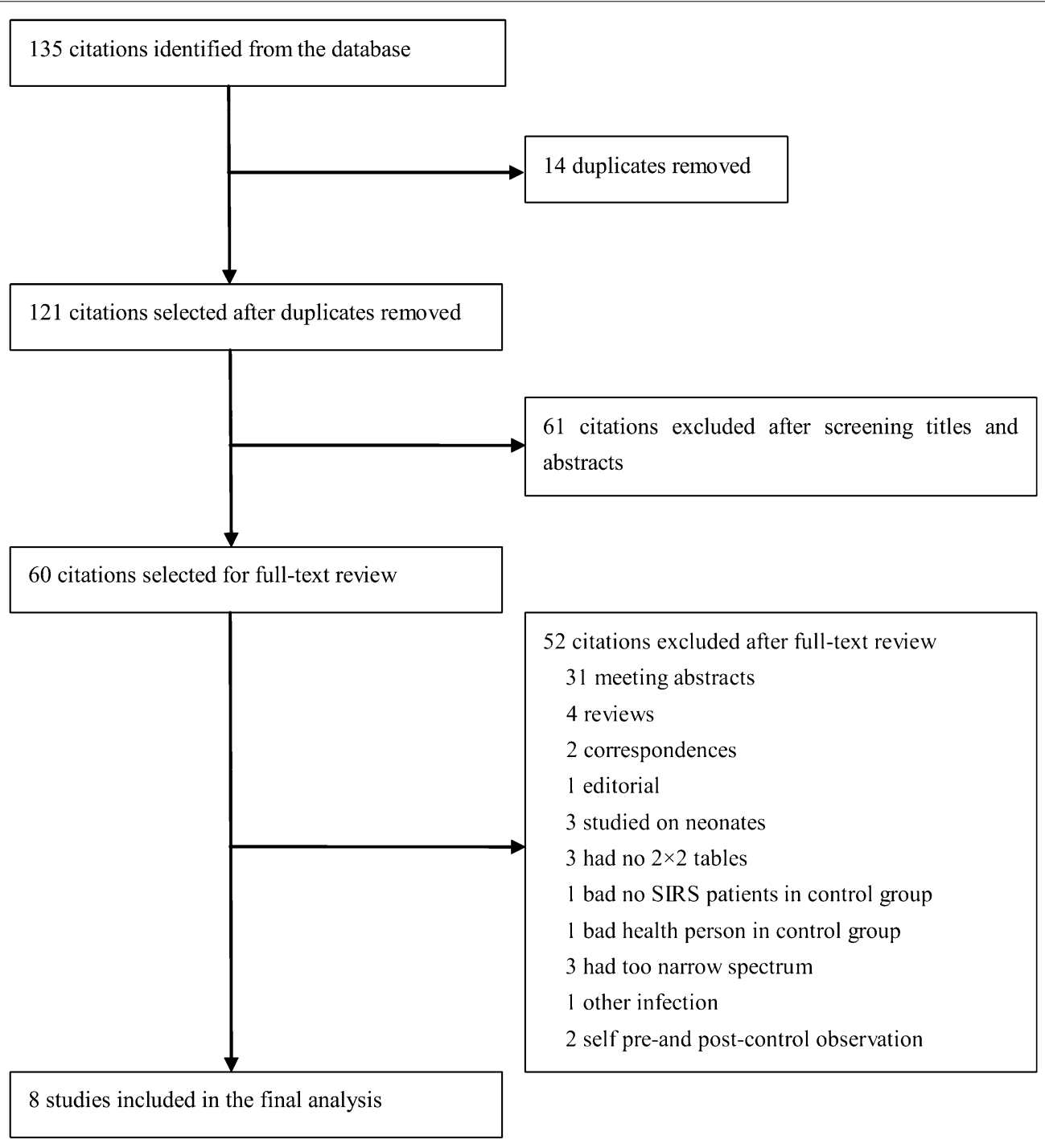

Fig. 1 Identification, inclusion, and exclusion of the studies

The SROC of presepsin is shown in Fig. 3, generating an AUC of $0.8598\left(Q^{*}=0.7906\right)$. A random effects model was used to calculate the pooled mean difference and the $95 \%$ confidence interval. The pooled diagnostic odds ratio of the eight studies was 14.25 (95\% CI 8.66-23.42). The pooled sensitivity and pooled specificity were 0.77 (95\% CI 0.75-0.80) and 0.73 (95\% CI 0.69-0.77), respectively (Fig. 4). The pooled positive LR and pooled negative LR were 3.11 (95 \% CI 2.16-4.50) and 0.22 (95 \% CI 0.160.32), respectively (Fig. 5). However, substantial degree of heterogeneity was observed in the summary estimates.

\section{Meta-regression}

Since obvious heterogeneity was observed, the meta-regression technique was used to explore the heterogeneity other than threshold effect. The result is shown in Table 2. A substantial heterogeneity caused by the measuring instrument was found, and the subgroup analysis was performed by restricting studies to a similar measuring instrument (PATHFAST, Mitsubishi Chemical Medience Corporation, Japan).

The analysis included four studies [7, 12, 42, 43],the SROC is shown in Fig. 6, and the AUC was 0.8946 $\left(Q^{*}=0.8255\right)$. The pooled sensitivity (random-effect model) and pooled specificity (fixed-effect model) were 0.75 (95 \% CI $0.72-0.78$ ) and 0.84 (95\% CI 0.79-0.88), respectively. The pooled positive LR (fixed-effect model) and pooled negative LR (random-effect model) were 4.82 (95\% CI 3.69-6.30) and 0.18 (95\% CI 0.09-0.35), respectively. The pooled diagnostic odds ratio was 18.57 (95\% 


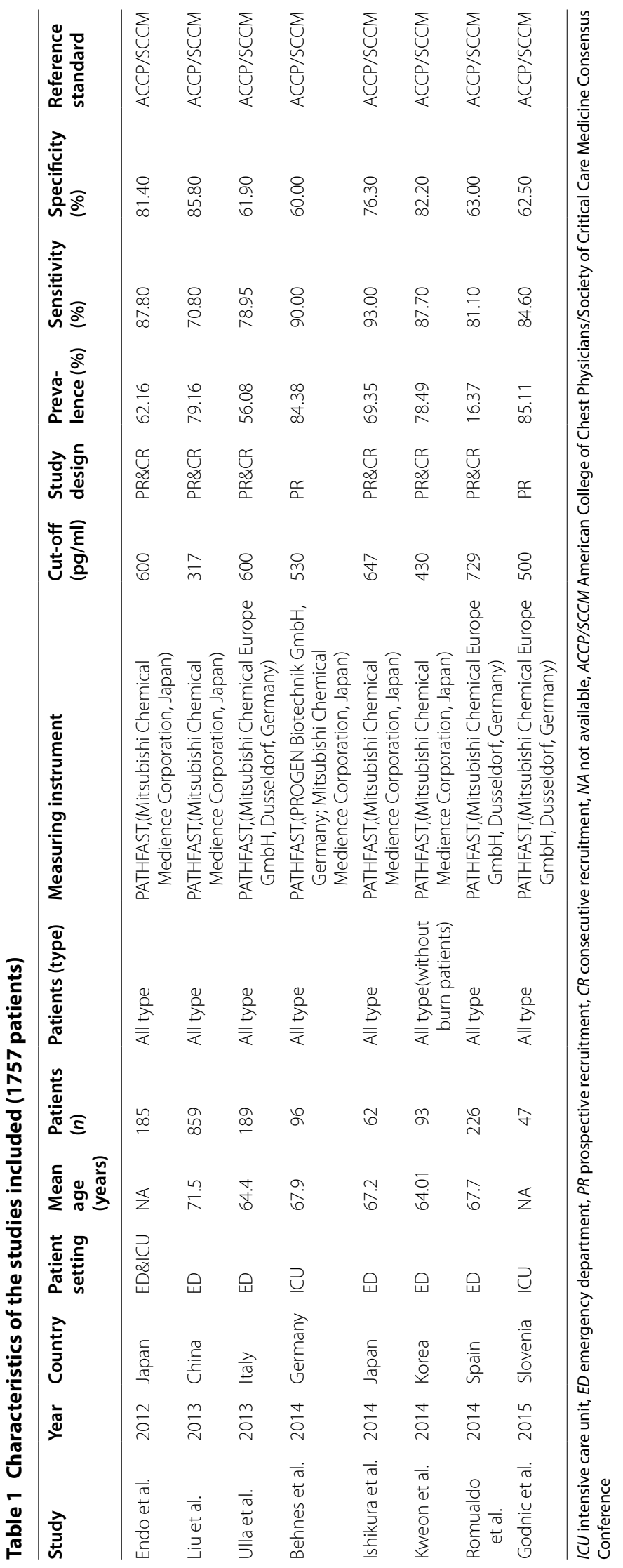




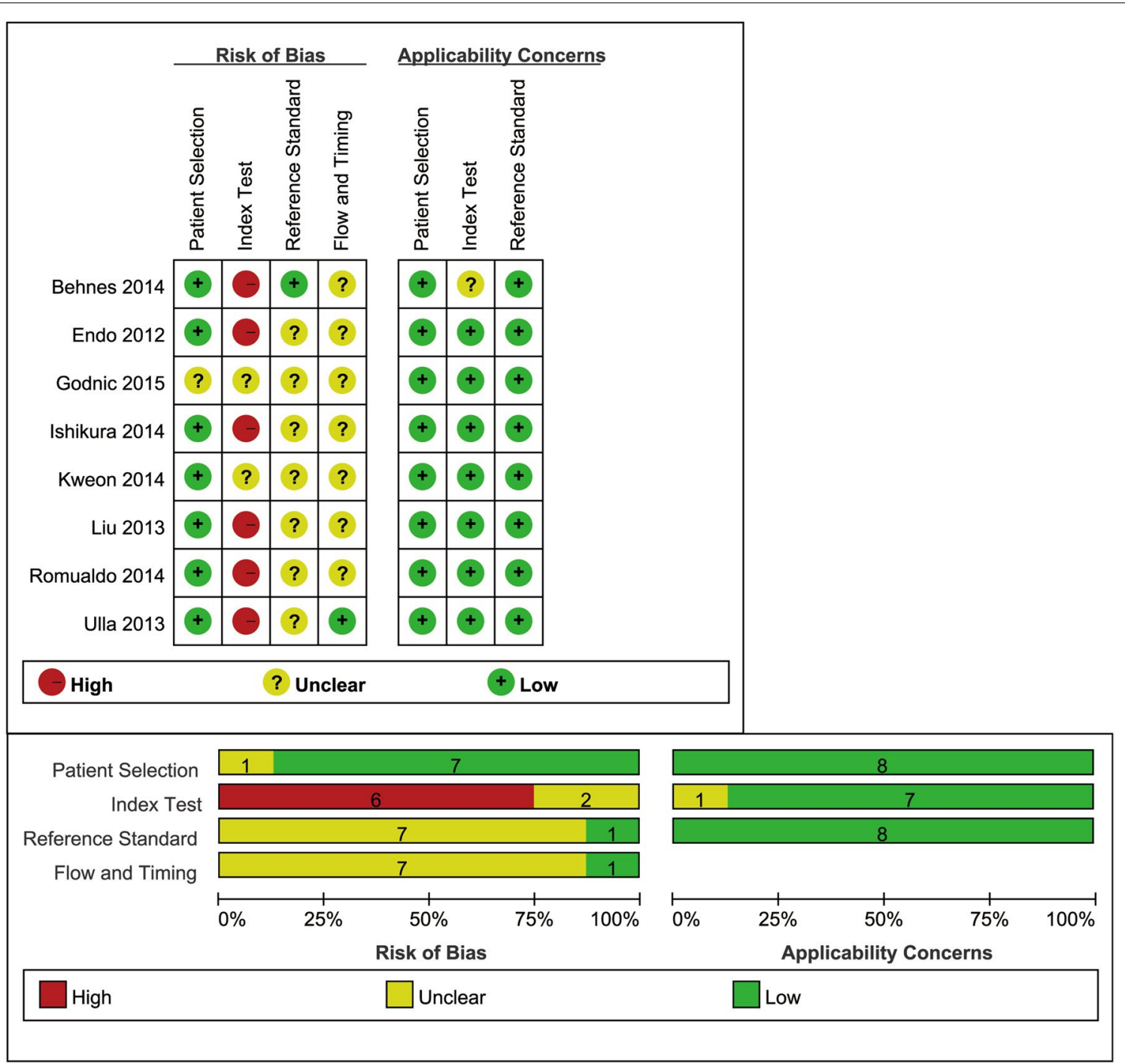

Fig. 2 Risk of bias and applicability concerns

CI 12.98-26.56). The result is shown in Table 3. However, there was still heterogeneity in pooled sensitivity and pooled negative LR $\left(I^{2}=91.4 \% \& I^{2}=86.8 \%\right)$.

\section{Subgroup analysis}

By observing the forest plots, the studies by Liu et al. and Endo et al. were found to be outliers and may account for most of the heterogeneity. After excluding these two studies, the heterogeneity diminished significantly. Subgroup analysis based on the rest of studies was performed with a fixed effects model, and the results are shown in Table 4 . The pooled sensitivity, specificity, positive LR, negative LR, and diagnostic OR were 0.85 (95\% CI 0.810.89), 0.65 (95\% CI 0.59-0.70), 2.46 (95\% CI 2.03-2.98), 0.25 (95 \% CI 0.19-0.33), and 9.47 (95\% CI 6.38-14.05), respectively. The SROC of these six studies are shown in Fig. 7 and the AUC was $0.8213\left(Q^{*}=0.7547\right)$.

\section{Publication bias exploration}

We used the Egger's regression model to detect the publication bias, and the result is shown in Fig. 8. The Deeks' funnel plot did not show significant asymmetry $(P=0.755)$, indicating that there was no significant publication bias in this meta-analysis. 


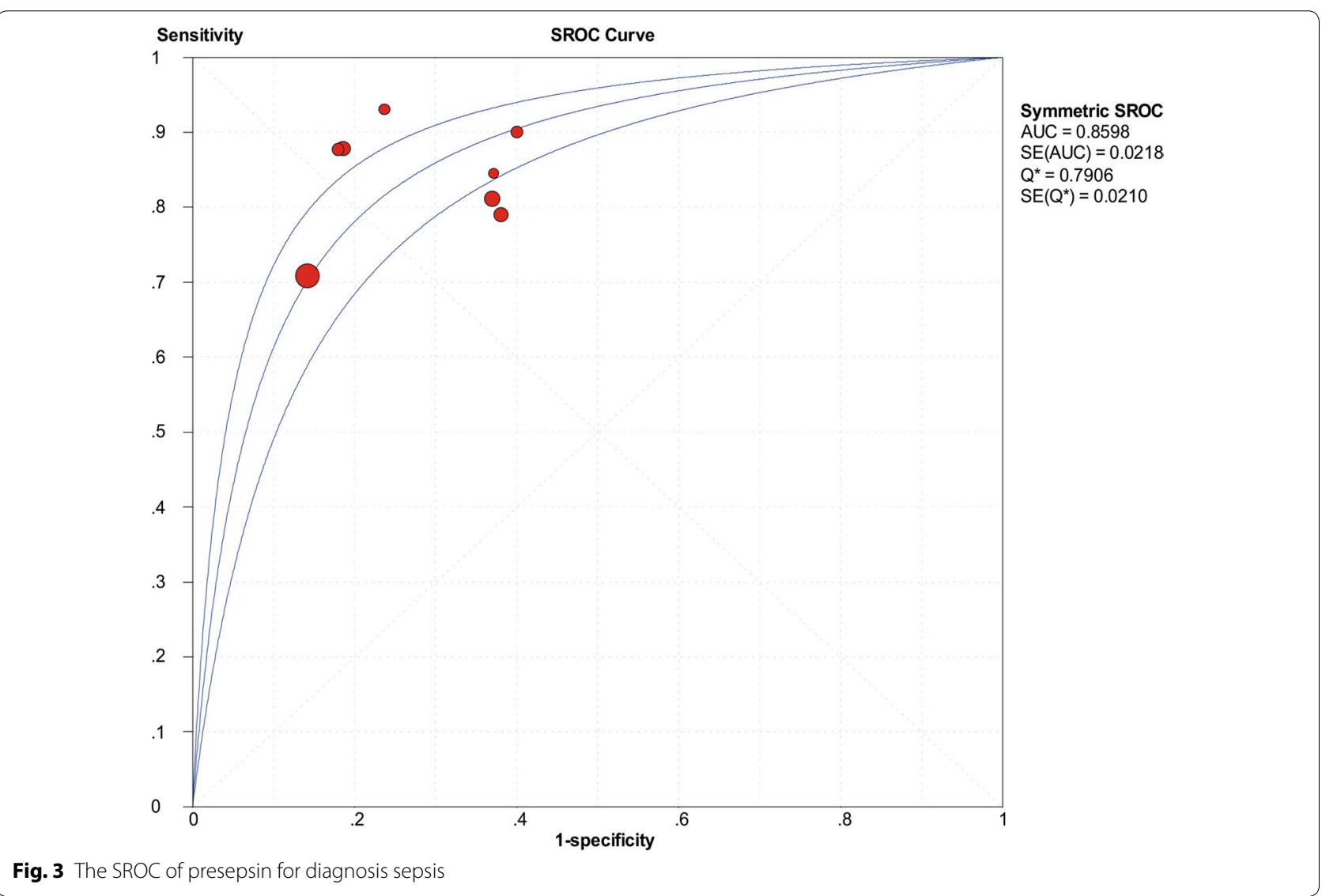

\section{Discussion}

The present systematic review and meta-analysis explored the accuracy of presepsin for the diagnosis of sepsis from SIRS patients. And the results showed that the biomarker presepsin had the acceptable pooled sensitivity (0.77) and pooled specificity (0.73). Accordingly, the AUC was 0.8598 , indicating that the presepsin had a moderate diagnostic efficiency.

Sepsis causes millions of deaths globally each year and occurs in 1-2 \% of all hospitalizations in the United States, affecting at least 750,000 persons and costing $\$ 17$ billion per year to treat $[1,2]$. As the high morbidity and mortality of sepsis, early diagnosis and treatment are essential to improve the prognosis of these patients [5]. However, due to the existence of other non-infectious causes of systemic inflammation (SIRS), it is critical to find a reliable biomarker to differentiate sepsis from SIRS at the early stage.

Various biological markers such as PCT, CRP, interleukins, and myeloid cells expressing triggering receptor-1 (TREM-1) have been reported as biomarkers in diagnosis with sepsis [47-49]. However, their clinical values are still controversial and uncertain except PCT. Meanwhile, the gold standard microbiological culture, which is used to distinguish infectious diseases from non-infectious conditions, lacks sensitivity and specificity, and there is often a substantial time delay [7].

As a new and promising biomarker, presepsin was first found in 2004 and has been revealed to be with superior diagnostic capacity when compared with other conventional diagnostic markers [11, 12]. Presepsin is a $13-\mathrm{kDa}$ protein that is a fragment of CD14 with 


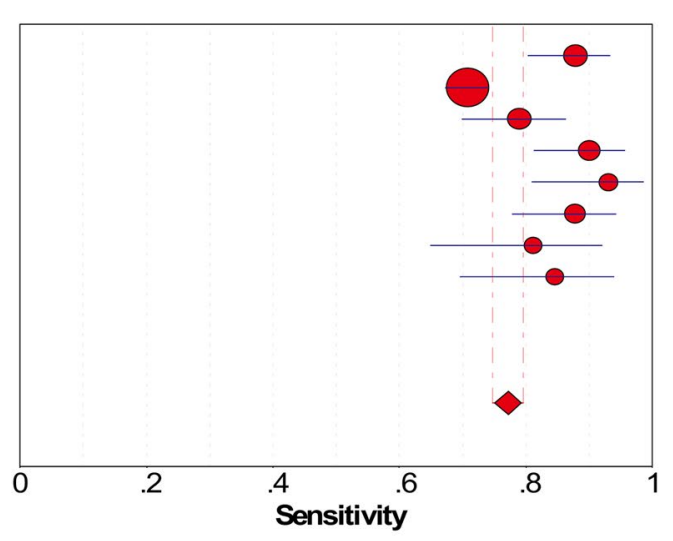

Endo et al

Liu et al

Ulla et al

Behnes et al

Ishikura et al

Kueon et al

Romualdo et al

Godnic et al

Pooled Sensitivity $=0.77$ (0.75 to 0.80$)$

Chi-square $=47.31 ; \mathrm{df}=7(p=0.0000)$

Inconsistency (I-square) $=85.2 \%$

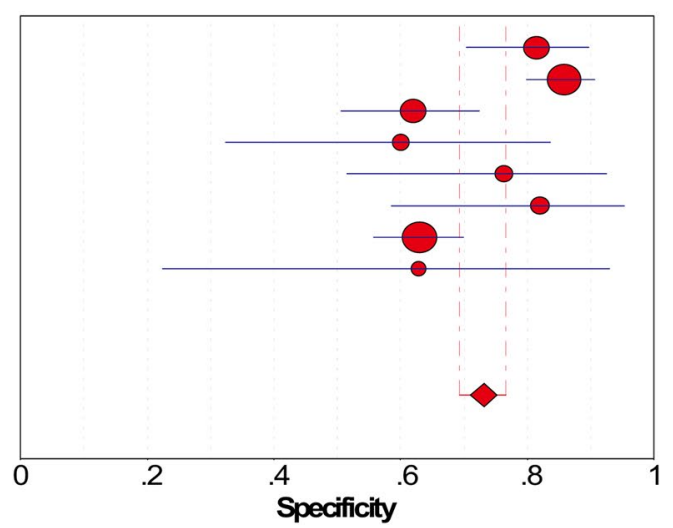

Endo et al

Liu et al

Ulla et al

Behnes et al

Ishikura et al

Kweonet al

Romualdo et al

Godnic et al

Pooled Specificity $=0.73$ (0.69 to 0.77 )

Chi-square $=36.01 ; d f=7(p=0.0000)$

Inconsistency (I-square) $=80.6 \%$

Fig. 4 Forest plot of the pooled sensitivity and specificity

truncated N-terminal, the receptor for LPS/LBP complexes. It was identified as a protein whose levels increase specifically in the blood of sepsis patients. Vodnik et al. found that presepsin values were significantly higher in patients with sepsis $(1508.3 \pm 866.6 \mathrm{pg} / \mathrm{mL})$ than the SIRS $(430.0 \pm 141.33 \mathrm{pg} / \mathrm{mL})$ group $(P<0.0001)$ [38]. However, the results of few studies showed considerable differences, such as in the sensitivity range (0.71-0.93) and in the specificity range $(0.60-0.86)$. Therefore, it is important for a meta-analysis to evaluate the value of a single laboratory test to identify patients at increased risk of sepsis from independent studies.

The meta-analysis presented above showed no threshold effect and significant publication bias. In addition, meta-regression was performed and a substantial heterogeneity caused by the measuring instrument was found. Therefore, subgroup analysis was performed by restricting studies to a similar measuring instrument (PATHFAST, Mitsubishi Chemical Medience Corporation, Japan); however, there were still heterogeneity in pooled sensitivity and pooled negative LR $\left(I^{2}=91.4 \% \& I^{2}=86.8 \%\right)$. The forest plots implied that the study by Liu et al. has the lowest sensitivity and the highest specificity. After excluding this study, heterogeneity still existed in pooled specificity $\left(I^{2}=53.7 \%\right)$ and pooled positive LR $\left(I^{2}=60.7 \%\right)$. The results in Fig. 4 showed that the study by Endo et al. may account for most of the heterogeneity in pooled specificity $\left(I^{2}=53.7 \%\right)$ and pooled positive LR. Statistical pooling of the summary estimates was made after 


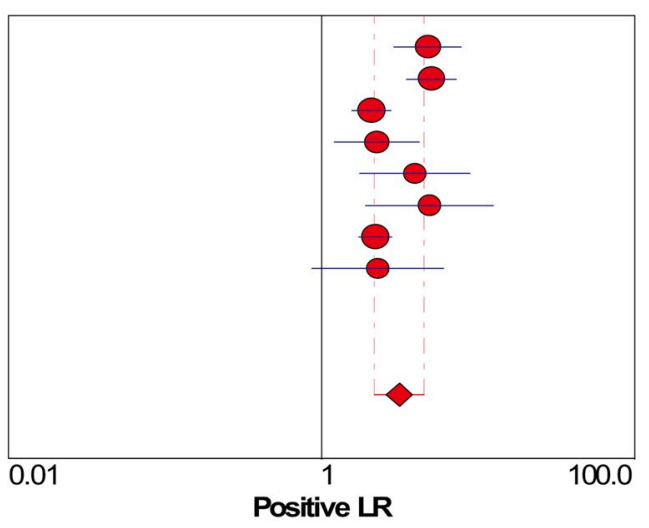

Endo et al

Liu et al

Ula et al

Behnes et al

Ishikura et al

Kweon et al

Romualdo et al

Godnic et al

RandomEffects Mbdel

Pooled Positive LR = 3.11 ( 2.16 to 4.50$)$

Cochran-Q = 33.55; df = $7(p=0.0000)$

Inconsistency (I-square) $=79.1 \%$

Tau-squared $=0.1935$

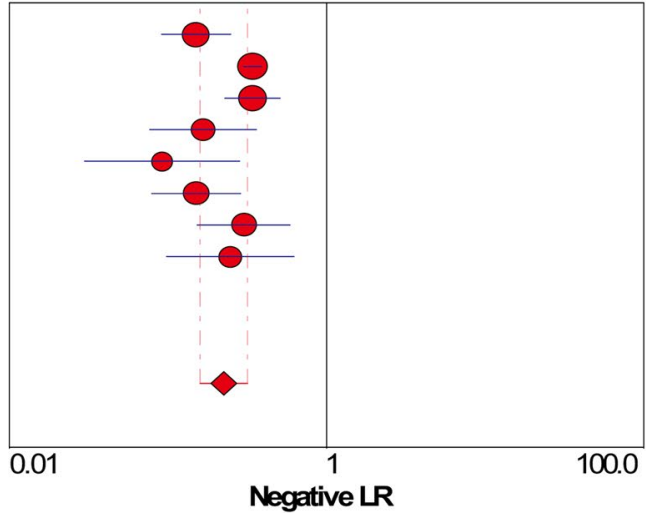

Endo et al

Liuet al

Ulla et al

Behnes et al

Ishikura et al

Kweon et al

Romualdo et al

Godnic et al
Positive LR (95\% Cl)

$4.73(2.88-7.76)$

$4.99 \quad(3.47-7.18)$

$2.07(1.55-2.78)$

$2.25(1.21-4.20)$

$3.93(1.75-8.84)$

$4.87(1.90-12.46)$

$2.19(1.72-2.79)$

$2.28(0.86-6.02)$

Random Effects Model

Pooled Negative LR $=0.22$ (0.16 to 0.32 )

Cochran $Q=24.37 ; d f=7(p=0.0010)$

Inoonsistency (I-square) $=71.3 \%$

Tau-squared $=0.1446$

Fig. 5 Forest plot of the pooled positive $L R$ and negative $L R$

Table 2 Meta-regression analysis for the possible sources of heterogeneity

\begin{tabular}{|c|c|c|c|c|c|}
\hline Variances & Coefficient standard & Standard error & $P$ value & RDOR & $95 \% \mathrm{Cl}$ \\
\hline \multicolumn{6}{|c|}{ Inverse variance weights 1} \\
\hline Cte & 3.344 & 0.9147 & 0.0353 & - & - \\
\hline S & 0.228 & 0.083 & 0.5130 & - & - \\
\hline Setting & 0.341 & 0.2697 & 0.2952 & 1.41 & $0.60-3.32$ \\
\hline Instrument & -0.945 & 0.4349 & 0.1180 & 0.39 & $0.10-1.55$ \\
\hline Cut-off & 0.046 & 0.0938 & 0.6558 & 1.05 & $0.78-1.41$ \\
\hline \multicolumn{6}{|c|}{ Inverse variance weights 2} \\
\hline Cte & 3.560 & 0.8027 & 0.0114 & - & - \\
\hline $\mathrm{S}$ & 0.282 & 0.2884 & 0.3838 & - & - \\
\hline Setting & 0.295 & 0.2531 & 0.3082 & 1.34 & $0.67-2.71$ \\
\hline Instrument & -0.978 & 0.4297 & 0.0851 & 0.38 & $0.11-1.24$ \\
\hline \multicolumn{6}{|c|}{ Inverse variance weights 3} \\
\hline Cte & 4.244 & 0.5481 & 0.0006 & - & - \\
\hline S & 0.458 & 0.2458 & 0.1215 & - & - \\
\hline Instrument & -1.200 & 0.3856 & 0.0265 & 0.30 & $0.11-0.81$ \\
\hline
\end{tabular}

The RDOR means the DOR for studies that lacked a particular methodologic feature divided by the DOR for studies without the flaw

-, not available, Cte constant term in the equation, RDOR relative diagnostic odds ratio, $S$ indicator of threshold

$P<0.05$ indicated the significant relationship between the characteristics of studies and the diagnostic odds ratio 


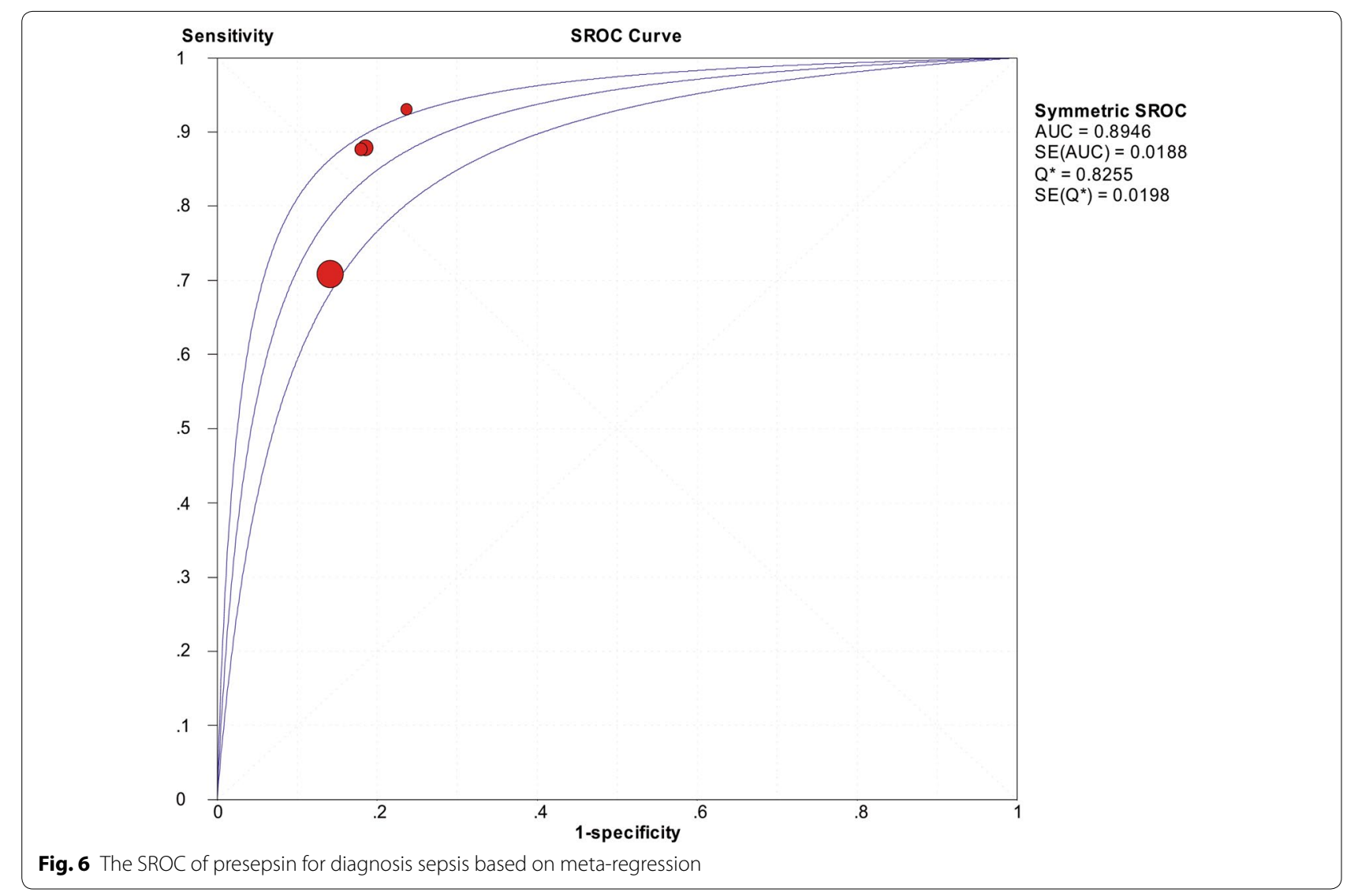

Table 3 Meta-regression analysis based on restricting studies to a similar measuring instrument

\begin{tabular}{lcrrr}
\hline Pooled results & Value & $\mathbf{9 5} \mathbf{\text { Cl}}$ & $\boldsymbol{P}$ value & $\boldsymbol{I}^{\mathbf{2}(\%)}$ \\
\hline Sensitivity & 0.75 & $0.72-0.78$ & 0.0000 & 91.4 \\
Specificity & 0.84 & $0.79-0.88$ & 0.6635 & 0.0 \\
Positive LR & 4.82 & $3.69-6.30$ & 0.9627 & 0.0 \\
Negative LR & 0.18 & $0.09-0.35$ & 0.0000 & 86.8 \\
Diagnostic OR & 18.57 & $12.98-26.560 .2197$ & 32.1 \\
\hline
\end{tabular}

Table 4 Subgroup analysis based on excluding outliers

\begin{tabular}{lllll}
\hline Pooled results & Value & $\mathbf{9 5} \mathbf{~ C l}$ & $\boldsymbol{P}$ value & $\boldsymbol{I}^{\mathbf{2}} \mathbf{( \% )}$ \\
\hline Sensitivity & 0.85 & $0.81-0.89$ & 0.1587 & 37.2 \\
Specificity & 0.65 & $0.59-0.70$ & 0.4438 & 0.0 \\
Positive LR & 2.46 & $2.03-2.98$ & 0.3472 & 10.7 \\
Negative LR & 0.25 & $0.19-0.33$ & 0.1079 & 44.6 \\
Diagnostic OR & 9.47 & $6.38-14.050 .1013$ & 45.7 \\
\hline
\end{tabular}

outliers excluded and the heterogeneity was eliminated, generating the acceptable pooled sensitivity $(0.85)$ and pooled specificity $(0.65)$.
The results of our study indicate moderate diagnostic performance of presepsin as a single test for diagnosing sepsis, which precludes recommendation for the routine use of sepsis as a screening or confirmatory test for sepsis. We are encouraged about the potential use of presepsin in combination with other clinical or laboratory markers in identifying high-risk patients, although we cannot make conclusions about this scenario because this was not an objective of the present study. The results of the study by Sargentini et al. confirmed the importance of monitoring a combination of several biomarkers in order to obtain a reliable diagnosis [35].

Because of its retrospective approach, any metaanalysis is prone to bias; however, we took several steps to minimize its impact. First, the selection of studies, extraction of data, and assessment of study quality were performed by two reviewers independently. Second, our study was adhered to the PRISMA statement. Third, only the ACCP/SCCM criterion was considered as our reference standard and studies were excluded if limited to restrictive subgroups. What is more, meta-regression and subgroup analysis were performed in this systematic review and meta-analysis, which made the result of the study more stable and reliable. 


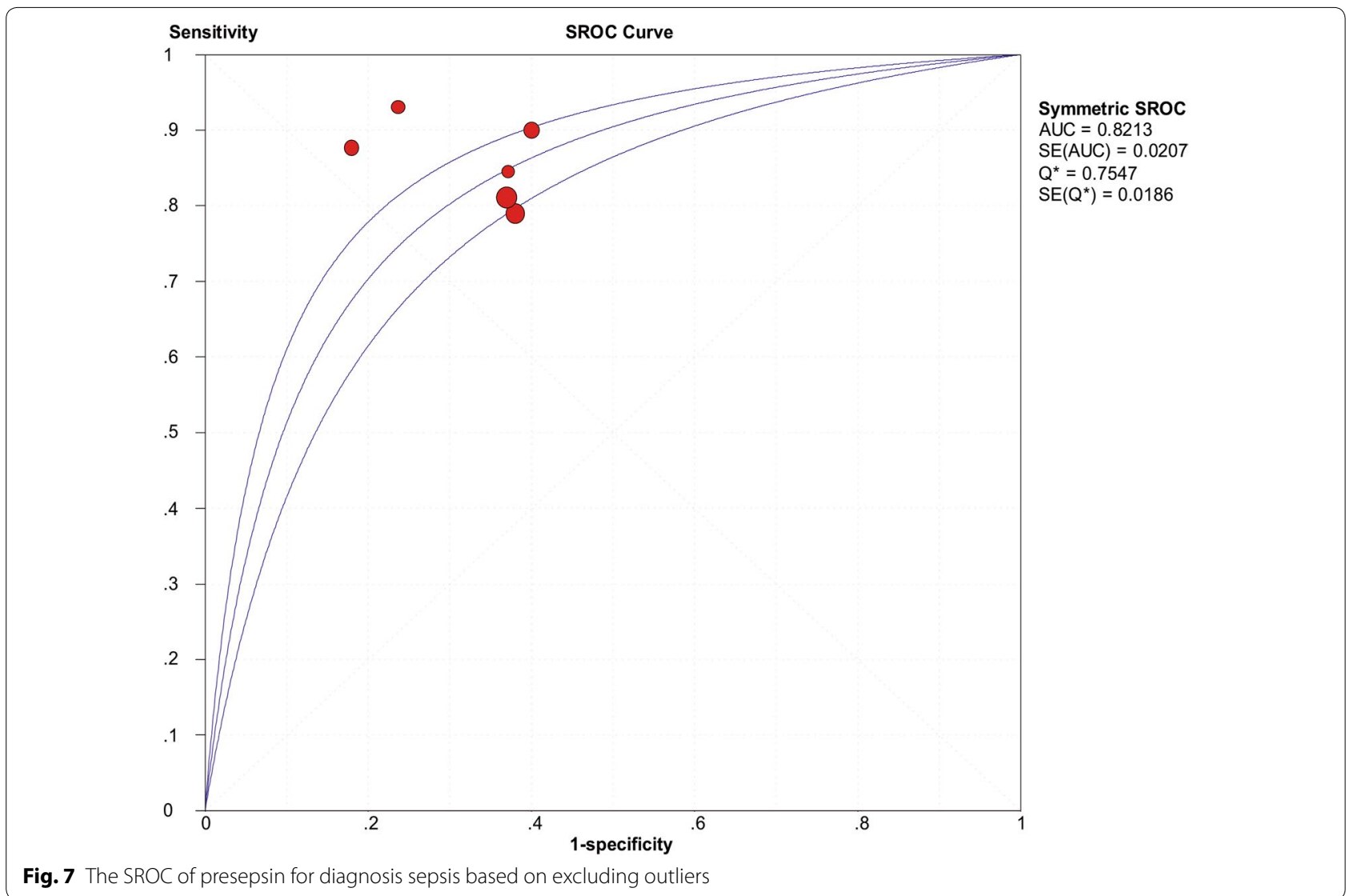

Fig. 7 The SROC of presepsin for diagnosis sepsis based on excluding outliers

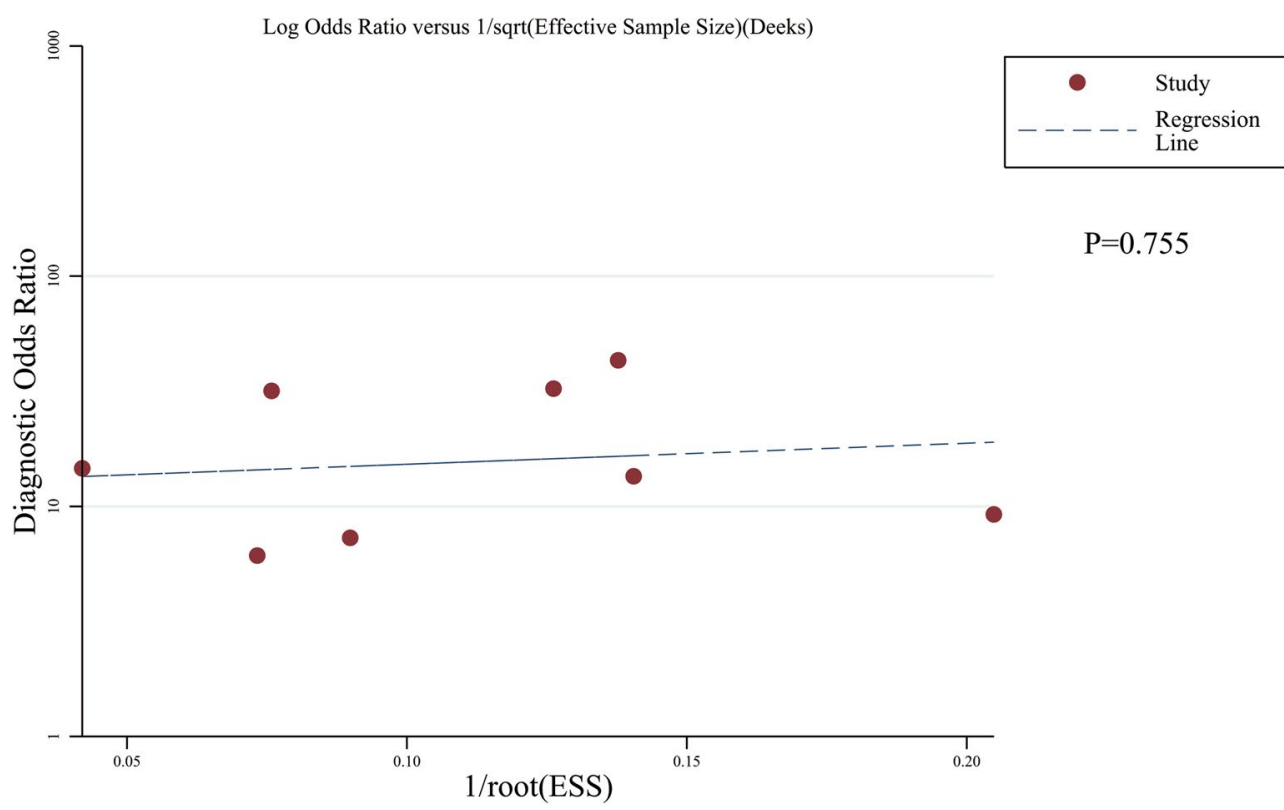

Fig. 8 Funnel plot of publication bias $(P=0.755)$ 
In addition, several limitations of this study should be put forward. First, studies included in our meta-analysis varied substantially in presepsin diagnostic criteria. Second, a limited number of studies were included in our meta-analysis. Third, the quality of all included studies varies substantially and might also influence the results of our studies. Therefore, further studies about presepsin for diagnostic assessment are need to be investigated, both in larger, multi-center studies and in prospective clinical studies, with sepsis patients without limited to restrictive subgroups.

\section{Conclusion}

Our meta-analysis found that the presepsin test has moderate diagnostic ability for the detection of sepsis. On the basis of diagnostic accuracy, our data suggest that, before widespread clinical use, further research is needed of the presepsin test as a diagnostic test used in isolation to include or exclude sepsis in emergency department. And presepsin in combination with other laboratory biomarkers in diagnosing sepsis may be the focus of future studies.

\begin{abstract}
Abbreviations
Presepsin, sCD14-ST: cluster-of-differentiation 14 subtypes; Cl: confidence interval; SROC: the summary receiver operating characteristic curve; AUC: the area under the receiver operating characteristic curve; MODS: multiple organ dysfunction; SIRS: systemic inflammatory response syndrome; LPS: lipopolysaccharide; LPB: lipopolysaccharide-binding proteins; PCT: procalcitonin; CRP: C-reactive protein; PRISMA: Preferred Reporting Items for Systematic Reviews and Meta-analyses Statement; EMBASE: Excerpta Medica database; MeSH: medical subject headings; ACCP/SCCM: American College of Chest Physicians/Society of Critical Care Medicine Consensus Conference; TP: true positive; FP: false positive; FN: false negative; TN: true negative; LR: likelihood ratio; OR: odds ratios; $I^{2}$ : inconsistency; TREM-1: myeloid cells expressing triggering receptor-1.
\end{abstract}

\section{Authors' contributions}

MZ conceived and designed the experiments. ZJZ, LBJ, and LGY performed the experiments. ZJZ, LBJ, LGY, and YZG analyzed the data. ZJZ, LBJ, LGY, YZG, and LPT contributed reagents/materials/analysis tools. ZJZ, LBJ, and LGY contributed to the writing of the manuscript. All authors read and approved the final manuscript.

\section{Acknowledgements}

This work was supported by the National key clinical specialist construction Programs of China, the National Key Technology R\&D Programme (2012BAl11B00), and the National Natural Science Foundation of China in 2013 (81372079).

\section{Competing interests}

The authors declare that they have no competing interests.

Received: 1 September 2015 Accepted: 13 November 2015 Published online: 08 December 2015

\section{References}

1. Chang HJ, Lynm C, Glass RM. JAMA patient page. Sepsis. JAMA. 2010;303(8):804. doi:10.1001/jama.303.8.804.
2. Martin GS, Mannino DM, Eaton S, Moss M. The epidemiology of sepsis in the United States from 1979 through 2000. N Engl J Med. 2003;348(16):1546-54. doi:10.1056/NEJMoa022139.

3. Linde-Zwirble WT, Angus DC. Severe sepsis epidemiology: sampling, selection, and society. Crit Care. 2004;8(4):222-6. doi:10.1186/cc2917.

4. Dombrovskiy VY, Martin AA, Sunderram J, Paz HL. Rapid increase in hospitalization and mortality rates for severe sepsis in the United States: a trend analysis from 1993 to 2003. Crit Care Med. 2007;35(5):1244-50. doi:10.1097/01.ccm.0000261890.41311.e9.

5. Dellinger RP, Levy MM, Rhodes A, Annane D, Gerlach H, Opal SM, et al. Surviving Sepsis Campaign: international guidelines for management of severe sepsis and septic shock, 2012. Intensive Care Med. 2013;39(2):165228. doi:10.1007/s00134-012-2769-8.

6. Bone RC, Balk RA, Cerra FB, Dellinger RP, Fein AM, Knaus WA, Guidelines for the use of innovative therapies in sepsis, The ACCP/SCCM Consensus Conference Committee, et al. AmerDefinitions for sepsis and organ failure ican College of Chest Physicians/Society of Critical Care Medicine. Chest. 1992;101(6):1644-55

7. Endo S, Suzuki Y, Takahashi G, Shozushima T, Ishikura H, Murai A, et al. Usefulness of presepsin in the diagnosis of sepsis in a multicenter prospective study. J Infect Chemother. 2012;18(6):891-7. doi:10.1007/ s10156-012-0435-2.

8. Shirakawa K, Naitou K, Hirose J, Takahashi T, Furusako S. Presepsin (sCD14ST): development and evaluation of one-step ELISA with a new standard that is similar to the form of presepsin in septic patients. Clin Chem Lab Med CCLM/FESCC. 2011;49(5):937-9. doi:10.1515/cclm.2011.145.

9. Yaegashi Y, Shirakawa K, Sato N, Suzuki Y, Kojika M, Imai S, et al. Evaluation of a newly identified soluble CD14 subtype as a marker for sepsis. J Infect Chemother. 2005;11(5):234-8. doi:10.1007/s10156-005-0400-4.

10. Wright SD, Ramos RA, Tobias PS, Ulevitch RJ, Mathison JC. CD14, a receptor for complexes of lipopolysaccharide (LPS) and LPS binding protein. Science. 1990;249(4975):1431-3.

11. Chenevier-Gobeaux C, Trabattoni E, Roelens M, Borderie D, Claessens YE. Presepsin (sCD14-ST) in emergency department: the need for adapted threshold values? Clin Chim Acta. 2014;427:34-6. doi:10.1016/j. cca.2013.09.019.

12. Liu B, Chen $Y X$, Yin Q, Zhao YZ, Li CS. Diagnostic value and prognostic evaluation of Presepsin for sepsis in an emergency department. Crit Care. 2013;17(5):R244. doi:10.1186/cc13070.

13. Ulla M, Pizzolato E, Lucchiari M, Loiacono M, Soardo F, Forno D, et al. Diagnostic and prognostic value of presepsin in the management of sepsis in the emergency department: a multicenter prospective study. Crit Care. 2013;17(4):R168. doi:10.1186/cc12847.

14. Mearelli F, Fiotti N, Altamura N, Paoli I, Casarsa C, Ruscio M, et al. Procalcitonin, presepsin, pro-adrenomedullin, fibrin degradation products, and lactate in early diagnosis and prognosis of septic patients newly admitted to the intermediate care unit from the emergency department. Crit Care. 2013;17(Suppl 4):P17.

15. American College of Chest Physicians/Society of Critical Care. Medicine Consensus Conference: definitions for sepsis and organ failure and guidelines for the use of innovative therapies in sepsis. Crit Care Med. 1992;20(6):864-74.

16. Mokart D, Merlin M, Sannini A, Brun JP, Delpero JR, Houvenaeghel G, et al. Procalcitonin, interleukin 6 and systemic inflammatory response syndrome (SIRS): early markers of postoperative sepsis after major surgery. $\mathrm{Br}$ J Anaesth. 2005;94(6):767-73. doi:10.1093/bja/aei143.

17. Thompson SG, Sharp SJ. Explaining heterogeneity in meta-analysis: a comparison of methods. Stat Med. 1999;18(20):2693-708.

18. Moses LE, Shapiro D, Littenberg B. Combining independent studies of a diagnostic test into a summary ROC curve: data-analytic approaches and some additional considerations. Stat Med. 1993;12(14):1293-316.

19. Lijmer JG, Bossuyt PM, Heisterkamp SH. Exploring sources of heterogeneity in systematic reviews of diagnostic tests. Stat Med. 2002;21(11):152537. doi:10.1002/sim.1185.

20. Higgins JP, Thompson SG. Quantifying heterogeneity in a meta-analysis. Stat Med. 2002;21(11):1539-58. doi:10.1002/sim.1186.

21. Walter SD. Properties of the summary receiver operating characteristic (SROC) curve for diagnostic test data. Stat Med. 2002;21(9):1237-56. doi:10.1002/sim.1099. 
22. Zamora J, Abraira V, Muriel A, Khan K, Coomarasamy A. Meta-DiSc: a software for meta-analysis of test accuracy data. BMC Med Res Methodol. 2006;6:31. doi:10.1186/1471-2288-6-31.

23. Mallet-Coste T, Chenevier-Gobeaux C, Fissore-Magdelein C, Magdelein X, Brod F, Claessens YE. Presepsin (SCD14-ST), an emergent and promising biomarker of infection. Annales Francaises de Medecine d'Urgence. 2013;3(5):305-9.

24. Mussap M, Noto A, Fravega M, Fanos V. Soluble CD14 subtype presepsin (sCD14-ST) and lipopolysaccharide binding protein (LBP) in neonatal sepsis: new clinical and analytical perspectives for two old biomarkers. J Maternal-fetal Neonatal Med. 2011;24(Suppl 2):12-4. doi:10.3109/147670 58.2011.601923.

25. Pizzolato E, Ulla M, Galluzzo C, Lucchiari M, Manetta T, Lupia E, et al. Role of presepsin for the evaluation of sepsis in the emergency department. Clin Chem Lab Med CCLM/FESCC. 2014;52(10):1395-400. doi:10.1515/ cclm-2014-0199.

26. Zou Q, Wen W, Zhang XC. Presepsin as a novel sepsis biomarker. World Emerg Med. 2014;5(1):16-9. doi:10.5847/wjem.j.1920-8642.2014.01.002.

27. Rabensteiner J, Skvarc M, Hoenigl M, Osredkar J, Prueller F, Reichsoellner $M$, et al. Diagnostic and prognostic potential of presepsin in Emergency Department patients presenting with systemic inflammatory response syndrome. Journal Infect. 2014;69(6):627-30. doi:10.1016/j. jinf.2014.07.024.

28. Faix JD. Presepsin - the new kid on the sepsis block. Clin Biochem. 2014;47(7-8):503-4. doi:10.1016/j.clinbiochem.2014.04.014.

29. Kwiatkowska-Gruca M, Behrendt J, Sonsala A, Wisniewska-Ulfik D, Mazur B, Godula-Stuglik U. Presepsin (soluble CD14-ST) as a biomarker for sepsis in neonates. Pediatr Pol. 2013;88(5):392-7.

30. Poggi C, Bianconi T, Gozzini E, Generoso M, Dani C. Presepsin for the detection of late-onset sepsis in preterm newborns. Pediatrics. 2015:135(1):68-75. doi:10.1542/peds.2014-1755.

31. Mussap M, Puxeddu E, Burrai P, Noto A, Cibecchini F, Testa M, et al. Soluble CD14 subtype (sCD14-ST) presepsin in critically ill preterm newborns: preliminary reference ranges. J Matern Fetal Neonatal Med. 2012;25(Suppl 5):51-3. doi:10.3109/14767058.2012.717462.

32. Masson S, Caironi P, Fanizza C, Thomae R, Bernasconi R, Noto A, et al. Circulating presepsin (soluble CD14 subtype) as a marker of host response in patients with severe sepsis or septic shock: data from the multicenter, randomized ALBIOS trial. Intensive Care Med. 2015;41(1):12-20. doi:10.1007/s00134-014-3514-2.

33. Okamura Y, Yokoi H. Development of a point-of-care assay system for measurement of presepsin (sCD14-ST). Clin Chim Acta. 2011;412(2324):2157-61. doi:10.1016/j.cca.2011.07.024.

34. Nakamura $Y$, Ishikura $H$, Nishida $T$, Kawano $Y$, Yuge $R$, Ichiki $R$, et al. Usefulness of presepsin in the diagnosis of sepsis in patients with or without acute kidney injury. BMC Anesthesiol. 2014;14:88. doi:10.1186/1471-2253-14-88.

35. Sargentini V, Ceccarelli G, D'Alessandro M, Collepardo D, Morelli A, D'Egidio A, et al. Presepsin as a potential marker for bacterial infection relapse in critical care patients. A preliminary study. Clin Chem Lab Med CCLM/FESCC. 2015;53(4):567-73. doi:10.1515/cclm-2014-0119.

36. Novelli G, Morabito V, Ferretti G, Pugliese F, Ruberto F, Rossi M, et al. PATHFAST presepsin assay for early diagnosis of bacterial infections in surgical patients, Preliminary study. Transplantation. 2012;94:532.

37. Cakir Madenci O, Yakupoglu S, Benzonana N, Yucel N, Akbaba D, Orcun Kaptanagasi A. Evaluation of soluble CD14 subtype (presepsin) in burn sepsis. Burns. 2014;40(4):664-9. doi:10.1016/j.burns.2013.08.024.
38. Vodnik T, Kaljevic G, Tadic T, Majkic-Singh N. Presepsin (sCD14-ST) in preoperative diagnosis of abdominal sepsis. Clin Chem Lab Med CCLM/ FESCC. 2013;51(10):2053-62. doi:10.1515/cclm-2013-0061.

39. Palmiere C, Mussap M, Bardy D, Cibecchini F, Mangin P. Diagnostic value of soluble CD14 subtype (sCD14-ST) presepsin for the postmortem diagnosis of sepsis-related fatalities. Int J Legal Med. 2013;127(4):799-808. doi:10.1007/s00414-012-0804-5.

40. Shozushima T, Takahashi G, Matsumoto N, Kojika M, Okamura Y, Endo S. Usefulness of presepsin (SCD14-ST) measurements as a marker for the diagnosis and severity of sepsis that satisfied diagnostic criteria of systemic inflammatory response syndrome. J Infect Chemother. 2011;17(6):764-9. doi:10.1007/s10156-011-0254-x.

41. Takahashi G, Shibata S, Ishikura H, Miura M, Fukui Y, Inoue Y, et al. Presepsin in the prognosis of infectious diseases and diagnosis of infectious disseminated intravascular coagulation: a prospective, multicentre, observational study. Eur J Anaesthesiol. 2015;32(3):199-206. doi:10.1097/ eja.0000000000000178.

42. Ishikura H, Nishida T, Murai A, Nakamura Y, Irie Y, Tanaka J, et al. New diagnostic strategy for sepsis-induced disseminated intravascular coagulation: a prospective single-center observational study. Crit Care. 2014;18(1):R19. doi:10.1186/cc13700.

43. Kweon OJ, Choi JH, Park SK, Park AJ. Usefulness of presepsin (sCD14 subtype) measurements as a new marker for the diagnosis and prediction of disease severity of sepsis in the Korean population. J Crit Care. 2014;29(6):965-70. doi:10.1016/j.jcrc.2014.06.014.

44. Behnes M, Bertsch T, Lepiorz D, Lang S, Trinkmann F, Brueckmann M, et al. Diagnostic and prognostic utility of soluble CD 14 subtype (presepsin) for severe sepsis and septic shock during the first week of intensive care treatment. Crit Care. 2014;18(5):507. doi:10.1186/s13054-014-0507-z.

45. Romualdo LGDG, Torrella PE, Gonzalez MV, Sanchez RJ, Holgado AH, Freire AO, et al. Diagnostic accuracy of presepsin (soluble CD14 subtype) for prediction of bacteremia in patients with systemic inflammatory response syndrome in the Emergency Department. Clin Biochem. 2014:47(7-8):505-8.

46. Godnic M, Stubljar D, Skvarc M, Jukic T. Diagnostic and prognostic value of sCD14-ST-presepsin for patients admitted to hospital intensive care unit (ICU). Wien Klin Wochenschr. 2015;127(13-14):521-7. doi:10.1007/ s00508-015-0719-5.

47. Harbarth S, Holeckova K, Froidevaux C, Pittet D, Ricou B, Grau GE, et al. Diagnostic value of procalcitonin, interleukin-6, and interleukin-8 in critically ill patients admitted with suspected sepsis. Am J Respir Crit Care Med. 2001;164(3):396-402. doi:10.1164/ajrccm.164.3.2009052.

48. Simon L, Gauvin F, Amre DK, Saint-Louis P, Lacroix J. Serum procalcitonin and C-reactive protein levels as markers of bacterial infection: a systematic review and meta-analysis. Clin Infect Dis. 2004;39(2):206-17. doi:10.1086/421997.

49. Wu Y, Wang F, Fan X, Bao R, Bo L, Li J, et al. Accuracy of plasma sTREM-1 for sepsis diagnosis in systemic inflammatory patients: a systematic review and meta-analysis. Crit Care. 2012;16(6):R229. doi:10.1186/cc11884.

\section{Submit your manuscript to a SpringerOpen ${ }^{\odot}$ journal and benefit from:}

- Convenient online submission

- Rigorous peer review

- Immediate publication on acceptance

- Open access: articles freely available online

- High visibility within the field

- Retaining the copyright to your article

Submit your next manuscript at $>$ springeropen.com 